\title{
UPPER BOUNDS FOR NORMS OF PRODUCTS OF BINOMIALS
}

\section{MIHAI CIPU}

\begin{abstract}
This paper deals with the problem of finding the least length of a product of $n$ binomials. A theorem of R. Maltby has shown that the problem is algorithmically solvable for any fixed $n$. Here, a different proof is presented for this result, and yields improved complexity. The author reports the results of computations of the upper bounds on the least length or Euclidean norm of a product of binomials.
\end{abstract}

\section{Introduction}

The classic Prouhet-Tarry-Escott problem is to find two disjoint multisets of $n$ integers such that the first $k<n$ fundamental symmetric polynomials take the same values when evaluated on the chosen multisets. This problem of 'equal sums of like powers' has a long history; a good source on recent work prompted by the Prouhet-Tarry-Escott problem is [7].

It is easy to find equivalent properties, as follows.

Proposition 1.1. Let $u_{1}, u_{2}, \ldots, u_{n}$ and $v_{1}, v_{2}, \ldots, v_{n}$ be integers with $u_{i} \neq v_{j}$ for all $1 \leqslant i, j \leqslant n$. For $1 \leqslant k<n$, the following statements are equivalent.

(i) $\sum_{i=1}^{n} u_{i}^{t}=\sum_{i=1}^{n} v_{i}^{t}$ for all $t, 1 \leqslant t \leqslant k$.

(ii) The polynomial $\prod_{i=1}^{n}\left(X-u_{i}\right)-\prod_{i=1}^{n}\left(X-v_{i}\right)$ has degree at most $n-k-1$.

(iii) $(X-1)^{k+1}$ divides $\sum_{i=1}^{n}\left(X^{u_{i}}-X^{v_{i}}\right)$.

An obvious way to fulfil condition (iii) is to have

$$
\sum_{i=1}^{n}\left(X^{u_{i}}-X^{v_{i}}\right)=X^{a} \prod_{i=1}^{k+1}\left(1-X^{a_{i}}\right)
$$

for suitable integers $a$ and $a_{i}$.

As early as 1851, Prouhet found that for any $k$ there is a solution with sufficiently large $n$. The most difficult situation, and thus the most interesting one, appears for $k=n-1$.

In order to describe a procedure that is instrumental in solving the Prouhet-Tarry-Escott problem for certain values of $k$ and $n$, we recall that the length or $l_{1}$-norm $L(P)$ of a polynomial $P$ is the sum of the absolute values of its coefficients. One can easily show that any polynomial $P$ that is the product of binomials $X^{u}-X^{v}$ gives rise to a solution to the Prouhet-Tarry-Escott problem, with $k$ one less than the number of binomials and $n$ equal to half of the length of the product.

Research partially supported by the EURROMMAT Programme, contract No. ICA1-CT-2000-70022 with the European Commission.

Received 19 June 2003, revised 16 December 2003; published 8 March 2004.

2000 Mathematics Subject Classification 11B75

(C) 2004, Mihai Cipu 
Therefore, in connection with the Prouhet-Tarry-Escott problem, it is of some importance to find

$$
A_{1}(n):=\min \left\{L\left(\prod_{i=1}^{n}\left(1-X^{a_{i}}\right)\right): a_{1}, a_{2}, \ldots, a_{n} \text { are positive integers }\right\} .
$$

Similar questions can be asked for any other norm on the space of polynomials. In [13], Erdős and Szekeres prove that for the sequence

$$
A_{\infty}(n):=\min \left\{\left\|\prod_{i=1}^{n}\left(1-X^{a_{i}}\right)\right\|_{\infty}: a_{1}, a_{2}, \ldots, a_{n} \text { are positive integers }\right\},
$$

where

$$
\left\|\sum_{i=0}^{d} c_{i} X^{i}\right\|_{\infty}:=\sup \left\{\left|\sum_{i=0}^{d} a_{i} z^{i}\right|: z \in \mathbb{C},|z|=1\right\},
$$

one has $\lim _{n \rightarrow \infty} A_{\infty}(n)^{1 / n}=1$. Maltby [18] considers the analogous quantity defined for the Euclidean norm:

$$
A_{2}(n):=\min \left\{\left\|\prod_{i=1}^{n}\left(1-X^{a_{i}}\right)\right\|_{2}: a_{1}, a_{2}, \ldots, a_{n} \text { are positive integers }\right\},
$$

with

$$
\left\|\sum_{i=0}^{d} c_{i} X^{i}\right\|_{2}:=\left(\sum_{i=0}^{d}\left|c_{i}\right|^{2}\right)^{1 / 2} .
$$

The values $A_{1}(n), A_{2}(n)$ and $A_{\infty}(n)$ are constrained by the well-known inequalities

$$
\frac{L(P)}{\sqrt{\operatorname{deg}(P)+1}} \leqslant\|P\|_{2} \leqslant\|P\|_{\infty} \leqslant L(P) \leqslant\|P\|_{2}^{2},
$$

which are valid for all polynomials $P$. So knowledge of one of these quantities produces a restriction on all the others. However, the Erdős-Szekeres problem is outside the scope of this paper. The ideas pointed out in the course of our approach to finding the value defined by relation (1) are completely different from those already used in dealing with the Erdős-Szekeres problem.

It has been known for a long time that $A_{1}(n) \geqslant 2 n$ for every natural number $n$. Erdôs and Szekeres proved that $A_{\infty}(n) \geqslant \sqrt{2 n}$, and the same lower bound is valid for $A_{2}(n)$. As far as we know, there have been no improvements on these old results. Furthermore, there still seems to be a lack of conjectures regarding the right order of magnitude of $A_{1}(n), A_{2}(n)$ or $A_{\infty}(n)$. Maltby [18] has devised an interesting construction to obtain upper bounds for $A_{1}(n)$ and $A_{2}(n)$. He considers a Weyl group $W$ associated to a root system of rank $t$, and expresses the positive roots $r_{i}$ in terms of the simple roots $\pi_{j}$ :

$$
r_{i}=\sum_{j=1}^{t} p_{i j} \pi_{j} \quad \text { for suitable positive integers } p_{i j} .
$$

Substituting arbitrary natural numbers $d_{1}, d_{2}, \ldots, d_{t}$ for the simple roots, one gets the pure product $P=\prod_{i=1}^{n}\left(1-X^{a_{i}}\right)$, corresponding to the exponents $a_{i}=\sum_{j=1}^{t} p_{i j} d_{j}$. Maltby proves that the length of the polynomial obtained in this way is bounded from above by the cardinality card $(W)$ of the group $W$, and that for suitable $d_{j}$ one also has $\|P\|_{2}^{2}=\operatorname{card}(W)$. 
This approach is appealing because of the flexibility in the choice of the values of $d_{j}$. It provides asymptotic upper bounds for the values $A_{1}(n)$ and $A_{2}(n)$ of the form

$$
A_{1}(n)<\sqrt{n}^{\sqrt{n}}, \quad A_{2}(n)<\sqrt{n}^{\sqrt{n} / 2},
$$

along with constructions of polynomials for which these bounds hold. However, it also has a very serious drawback: the order of the Weyl group can be very large in comparison with $t$. For instance, one has $n=t^{2}$ and $\operatorname{card}(W)=2^{t} \cdot t$ ! for the group $W$ of type $B_{t}$. Therefore, the bound for $A_{1}(n)$ thus obtained is no better than that inferred by (3) from an example previously given by Atkinson [2] and Dobrowolski [12], who used pure products generated by Vandermonde determinants to show that

$$
A_{\infty}(n)<(\sqrt{2 n})^{\sqrt{n / 2}} .
$$

Erdős and Szekeres put forward the conjecture that $A_{\infty}(n) \leqslant \exp \left(n^{c}\right)$ for some positive constant $c$. The best result in this direction is due to Belov and Konyagin [3]: $A_{\infty}(n)<$ $\exp \left(c(\ln n)^{4}\right)$ for a certain constant $c$, improving on earlier work of Odlyzko [20] and Kolountzakis [15].

Besides lower and upper bounds on $A_{1}(n)$, one may find in the literature the exact values for several small positive integers $n$. The only values known before 1994 were $A_{1}(n)=2 n$ for $n \leqslant 6$ and $n=8$. In that year, P. Borwein and C. Ingalls [7] reported the results of various computations for $n$ at most 100. Using various greedy algorithms to find the exponents $a_{i}$, they produced upper bounds $B(n)$ for $A_{1}(n)$. On the basis of extensive computations, they conjectured that $A_{1}(7)=16$. This was later confirmed by Maltby [17]. Combining extensive computer searches with theoretical results, Maltby also obtained $A_{1}(9)=20$ and $A_{1}(10)=24$. However, his main contribution was to show that a finite amount of computation is sufficient to find the value $A_{1}(n)$ for any given $n$.

The first purpose of this paper is to give a proof of the following result.

THEOREM 1.2. For a given $n$, a minimum-length polynomial of the form $\prod_{i=1}^{n}\left(1-X^{b_{i}}\right)$ occurs with all $b_{i}$ having values of at most $(n-1)^{(n-1) / 2}$.

Maltby [16, 17] proved Theorem 1.2 with the weaker bound $n^{(n-1) / 2}(n-1)^{(n-1) / 4}$. In [11] it is shown that, having a length $K$ polynomial of the form $\prod_{i=1}^{n}\left(1-X^{a_{i}}\right)$, one can get a minimum-length polynomial of the form $\prod_{i=1}^{n}\left(1-X^{b_{i}}\right)$ by checking the length for all values of $b_{i}$ up to a certain value $M$ that depends on $n$ and also on $K$, for $a_{1}, \ldots, a_{n}$. Moreover, $M$ is adjusted dynamically during the execution of the algorithm.

As a consequence of the theorem above, we find that the most unsophisticated idea of trying all values of the exponents up to a certain bound $M$ and writing down the smallest length of the corresponding polynomials eventually gives the value of $A_{1}(n)$. In his Ph.D. thesis [16] (see also [17]), Maltby presents three algorithms for checking whether $A_{1}(n) \leqslant$ $K$ for any fixed $K$. The most sophisticated algorithm has been successful in establishing the complete list of exponents, yielding $A_{1}(n)$ for $n \leqslant 9$. Another purpose of this present paper is to present in Sections 3-5 several heuristic approaches that allow one to bound $A_{1}(n)$ from above for many values of $n$. Besides improving on previously published results, these bounds are interesting because, by using them, one can prove that the entries of the record exponent vectors must satisfy a number of divisibility conditions (see Section 6). Finally, we discuss the output of our searches. 


\section{Proof of Theorem 1.2}

All the approaches mentioned in [17] and [11] are based on the idea that finding the products of binomials with small lengths amounts to studying equalities among sums of subsets of a finite set of natural numbers. The idea is easily formalized, but requires some notation.

For $n$ a positive integer, denote $[n]:=\{1,2, \ldots, n\}$. The cardinality of a set $J$ is denoted by $|J|$. The following statement has an obvious proof.

LemMa 2.1. Let $a_{1}, a_{2}, \ldots, a_{n}$ be positive integers with sum $d$. For $t=0,1,2, \ldots, d$, let

$$
\begin{aligned}
\mathcal{E}_{t}:= & \left\{I \subseteq[n]:|I| \text { even, } \sum_{i \in I} a_{i}=t\right\} ; \\
\mathcal{O}_{t}:= & \left\{J \subseteq[n]:|J| \text { odd, } \sum_{j \in J} a_{j}=t\right\} ; \\
& \prod_{i=1}^{n}\left(1-X^{a_{i}}\right)=\sum_{j=0}^{d} c_{j} X^{j} .
\end{aligned}
$$

Then $c_{t}=\left|\mathcal{E}_{t}\right|-\left|\mathcal{O}_{t}\right|$ for $t=0,1,2, \ldots, d$.

For all $t, 0 \leqslant t \leqslant d, I \in \mathcal{E}_{t}$ and $J \in \mathcal{O}_{t}$, define $A_{1}(I, J)$ to be the $1 \times n$ matrix $\left(x_{1} x_{2} \ldots x_{n}\right)$, whose entries are given by $x_{i}=1$ for $i \in I \backslash J, x_{j}=-1$ for $j \in J \backslash I$, and zero otherwise. Build a matrix $A$ by putting together all the lines $A_{1}(I, J)$. The column vector $\mathbf{v}$ whose entries are the given natural numbers $a_{1}, a_{2}, \ldots, a_{n}$ satisfies $A \mathbf{v}=0$. It is easily seen that the rank $r$ of the matrix $A$ is less than $n$.

The next step in the proof consists of replacing the exponents $a_{i}$. To this end, Maltby uses the Bombieri-Vaaler [4] improvement of Siegel's lemma. We find an older result of Borosh more convenient. By [6], the homogeneous linear system of equations $A Y=0$ has a nontrivial solution $\mathbf{u}$ whose entries are natural numbers $b_{1}, b_{2}, \ldots, b_{n}$ at most

$$
B:=\max \{|\operatorname{det}(C)|: C \text { an } r \times r \text { submatrix of } A\} .
$$

One can show that $b_{i}>0$ for all $i \in[n]$; see [17, (3.2) and (3.3)].

Finally, we have to bound from above $B$. To this end, we apply the Nowosad-Tovar inequality [19]

$$
\sum_{i}\left|\lambda_{i}\right|^{2} \leqslant \sum_{i}\left(\sum_{j}\left|c_{i j}\right|^{2}\right)^{1 / 2}\left(\sum_{j}\left|c_{j i}\right|^{2}\right)^{1 / 2},
$$

where the $\lambda_{i}$ are the eigenvalues of an $r \times r$ matrix $C=\left(c_{i j}\right)$. In the case at hand, we have rank $C=r$ and all $c_{i j} \in\{-1,0,1\}$, so the right-hand side of the relation (4) is at most $r \cdot r$. For the left-hand side, we use the arithmetic mean-geometric mean inequality, which results in $\sum\left|\lambda_{i}\right|^{2} \geqslant r|\operatorname{det}(C)|^{2 / r}$. Hence,

$$
B \leqslant r^{r / 2} \leqslant(n-1)^{(n-1) / 2},
$$

which concludes the proof of Theorem 1.2.

The significance of this result is mainly theoretical: it shows that to find all possible lengths of the product of $n$ binomials, it suffices to check all the exponents below $(n-1)^{(n-1) / 2}$. This manner of finding $A_{1}(n)$ is clearly impractical for $n \geqslant 10$. Perhaps more useful than the result itself, is its proof. Presumably, by combining Borosh's theorem, 
the Nowosad-Tovar inequality and Maltby's second algorithm, one could find a practical way to obtain $A_{1}(n)$. This method seems unlikely to run out of cases to check for any $n$ for which $A_{1}(n)$ is not already known - at least in the computational environment to which we have access. We therefore examined several other ideas, whose outcomes are discussed in the next few sections.

Table 1: Published results.

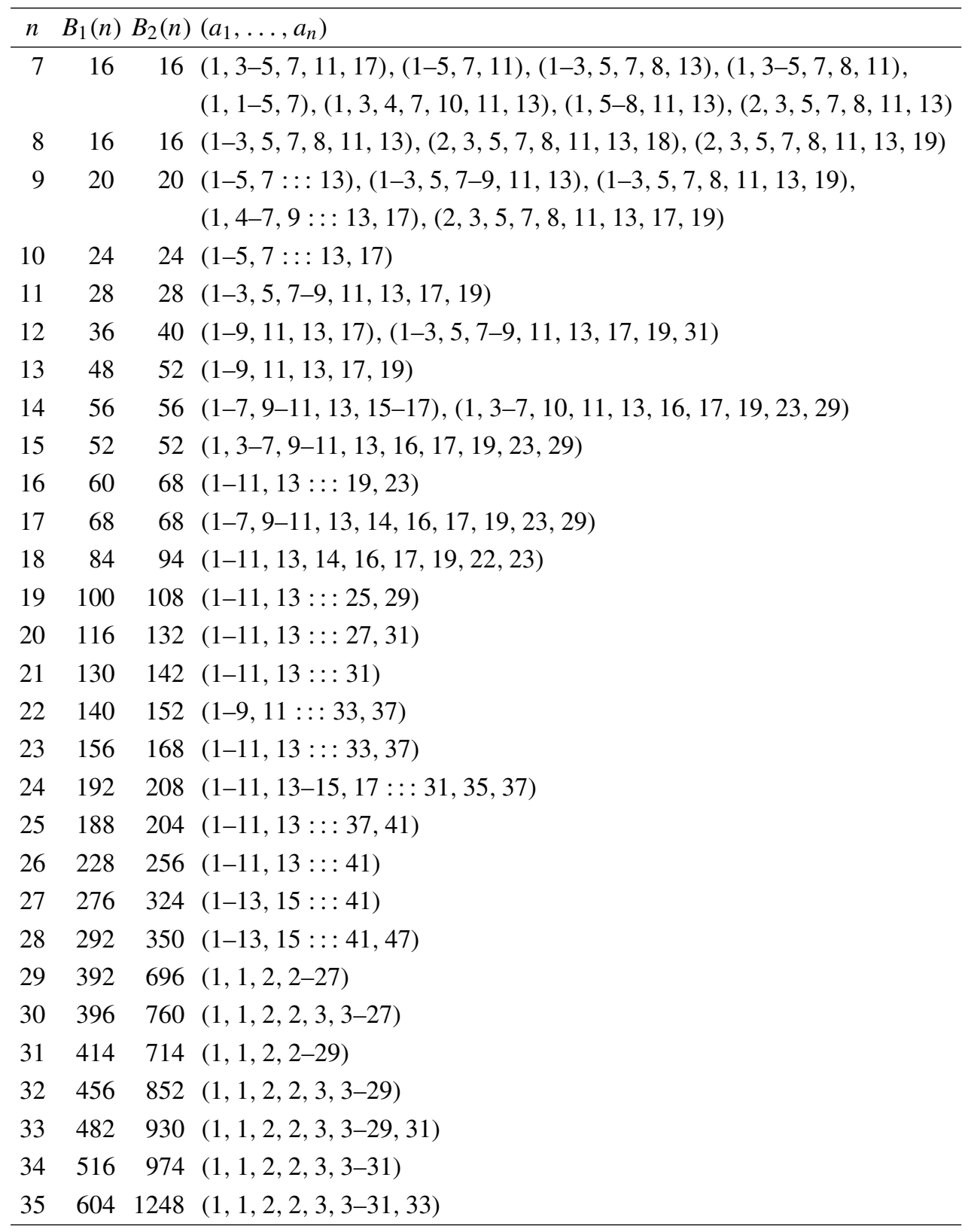




\section{A heuristic search}

The starting point of the work reported in this section is the information conveyed by results published in [7], [17] and [18]. Most of the computations have been performed by P. Borwein. For the reader's convenience, we reproduce some of the data in Table 1. The second and third columns show the tightest upper bounds for $A_{1}(n)$ and $A_{2}(n)^{2}$, respectively. The fourth column lists all the vectors for which it is known that the value appearing in the second column of the same line is attained. We use the notation ' $a-b$ ' as a shorthand for the numerical range $a, a+1, \ldots, b-1, b$. The double ellipsis ' $a::: b$ ' means that every odd number between the bounds appears exactly once.

It is apparent that a solution must have considerable additional structure. It is not so obvious how to put this observation in quantitative terms. From the available data, one notices some features of the record vectors giving $A_{1}(n)$ or the best upper bound currently known for it. Perhaps the most obvious ones are the following.

(1) $1 \leqslant a_{1} \leqslant 2$.

(2) $a_{3}=a_{1}+a_{2}$.

(3) There exists $k>3$ such that $a_{k}=a_{2}+a_{3}$.

(4) There exists one entry equal to 4 or 8.

The entries of the vectors yielding $A_{1}(n)$ are strikingly small, and it is not difficult to come up with a heuristic explanation of this observation. One way to make this vague remark specific is as follows.

(5) $a_{n}$ is at most the $n$th prime number.

Admittedly, stated in this way, property (5) is the most intriguing.

A further look at the results published by Borwein and Ingalls or by Maltby suggests that, at least for $n \geqslant 8$, one has the following properties.

(6) $A_{1}(n)$ strictly increases with $n$.

(7) $A_{1}(n)$ is a multiple of 4.

There are easily spotted entries for the upper bounds $B_{1}(n)$ in Table 1 that cast doubt on each of these properties. For instance, $B_{1}(14)>B_{1}(15)$ and $B_{1}(24)>B_{1}(25)$, while $B_{1}(21)=130$ is not a multiple of 4 , nor is $B_{1}(31)=414$, but we interpret this data as a hint that the values in the second column are far from being the desired $A_{1}(n)$. Moreover, Maltby [17] has shown that the length of a polynomial $P=\prod_{i=1}^{n}\left(1-X^{a_{i}}\right)$ is a multiple of 4 , provided that $n$ is even or $P$ has odd degree. However, no proof for property (7) is presently known.

Taking for granted the properties (1)-(5), one can incorporate them in a first procedure: simply keep substituting values for $a_{1}, a_{2}, \ldots, a_{n}$, and see what lengths result. The output of the computer program implementing this heuristic contains better upper bounds for $A_{1}(13)$, as well as new record vectors of length 12 ; see Table 2 . We mention that not all pure products corresponding to the vectors from the line $n=12$ have Euclidean norm $6=\sqrt{36}$.

Table 2: Output of the first heuristic search.

\begin{tabular}{cccl}
\hline$n$ & $B_{1}(n)$ & $B_{2}(n)$ & $\left(a_{1}, \ldots, a_{n}\right)$ \\
\hline 12 & 36 & 36 & $(1-5,7::: 19),(1-3,5-9,11,13,17,19)$, \\
& & & $(1-3,5,7-9,11,13,17,19,37),(1-3,5,7-9,11,13,17,19,47)$ \\
13 & 44 & 44 & $(1-5,7::: 13,16,17,19,23)$ \\
\hline
\end{tabular}


The last vector on the line $n=12$ is particularly interesting: it contains an entry greater than 37 , the twelfth prime number. Consequently, if property (5) turns out to be true, then $A_{1}(12) \leqslant 32$. Conversely, in order to reject heuristic (5), it would be sufficient to show that $A_{1}(12)=36$.

We conducted searches in a broader range than that allowed by condition (5) above. Even when $a_{n}$ was as large as the $(n+4)$ th prime number, there were no improvements. From the available data (see the tables above) one may conclude that the record vectors for $n \geqslant 8$ have distinct entries. By taking account of this observation, one may obtain a slight speed-up of the heuristic search.

Table 3: Output of the ascending search.

\begin{tabular}{|c|c|c|c|}
\hline$n$ & $B_{1}(n)$ & $B_{2}(n)$ & $\left(a_{1}, \ldots, a_{n}\right)$ \\
\hline 11 & 28 & 28 & $(1,2,5-9,11,13,17,19)$ \\
\hline 14 & 52 & 52 & $(1,2,5-9,11::: 19,20,25)$ \\
\hline 16 & & 64 & $(1,3-7,9-11,13,14,16,17,19,23,29)$ \\
\hline 21 & 130 & 130 & $(1-7,9-11,13,15-17,19::: 31)$ \\
\hline 27 & 262 & 314 & $(1-11,13::: 19,23::: 43,47)$ \\
\hline 28 & 280 & 336 & $(1-11,13::: 43,47)$ \\
\hline 29 & 316 & 348 & $(1-13,15::: 43,47)$ \\
\hline 30 & 368 & 430 & $(1-7,9-13,15-17,19::: 47)$ \\
\hline 31 & 360 & 412 & $(1-7,9-17,19::: 43,47,53)$ \\
\hline 34 & 460 & 546 & $(1-11,13-17,19,21,23-25,27::: 49,53)$ \\
\hline 34 & 460 & & $(1-11,13-15,17-19,21,23-25,27,29,31-33,35::: 49)$ \\
\hline 35 & 456 & 560 & $(1-11,13-17,19,21,23-25,27::: 53)$ \\
\hline 36 & 492 & 584 & $(1-17,19::: 53,59)$ \\
\hline 41 & 734 & 954 & $(1-17,19,21-23,25::: 61,67)$ \\
\hline 42 & 872 & & $(1-17,19-23,25::: 61,67)$ \\
\hline 42 & & 1240 & $(1-17,19,21-23,25::: 61,67,71)$ \\
\hline 43 & 902 & 1234 & $(1-19,21::: 63,67,73)$ \\
\hline 47 & 1232 & 1804 & $(1-21,23::: 69,73,79)$ \\
\hline 51 & 1632 & 2660 & $(1-21,23,25,27-29,31::: 75,79,85)$ \\
\hline 53 & 1596 & 2436 & $(1,2,2-23,25::: 79,85)$ \\
\hline 54 & 1712 & 2616 & $(1,2,2-21,23::: 85)$ \\
\hline 55 & 1728 & 2628 & $(1,2,2-23,25::: 83,89)$ \\
\hline 57 & 1896 & 3144 & $(1,2,2-27,29::: 83,89)$ \\
\hline 62 & 2456 & 4372 & $(1,2,2-27,29::: 65,65,67::: 91,101)$ \\
\hline 63 & 2796 & 5136 & $(1,2,2-25,27::: 97,103)$ \\
\hline
\end{tabular}




\section{Ascending search}

The search described in the previous section was based on properties seemingly shared by the entries in all the lines of Table 1 . Besides this horizontally driven search, a different approach can be taken. A closer look at the entries of the record vectors reveals strong similarities between neighbours in the last column. We concentrate on only one remark, as follows.

(8) The exponents yielding $B_{1}(n)$ are obtained by deleting one entry and inserting two entries in a record vector for $n-1$.

For instance, $A_{1}(10)$ is attained by a vector closely related to the last-but-one vector yielding $A_{1}(9)$ : we delete 6 and insert 2 and 3. The 'ancestor' is by no means unique: the same vector on the line $n=10$ can be generated by removing 8 and inserting 4 and 17 in the second vector on the line $n=9$.

The output of the search guided by properties (8) and (5) is given in Table 3 . One notices that now we have $B_{1}(14)=B_{1}(15)=52$. If these values are actually $A_{1}(14)$ and $A_{1}(15)$, then assumption (6) must be rejected. The new record vectors for $n=11$ and $n=14$ are particularly interesting: they are the only examples in disagreement with hypothesis (2). In view of Maltby's extensive computations, we conjecture that $A_{1}(11)=28$, and therefore that hypothesis (2) must be rejected.

The entry in the line $n=21$ was discovered by Borwein and Mossinghoff in their study of polynomials with height 1 and prescribed vanishing at 1; see [8]. It is also possible that other entries in Tables 2-4 were known to people working on related problems, but we could not locate any other results overlapping with ours, in the literature.

\section{Descending search}

There is an alternative possible way of making use of the observation formalized in condition (8). That is, we go in the opposite direction: we start with a vector of length $n$, remove two of its entries and insert only one value that is subject to restriction (5). This idea is worth pursuing, as witnessed by Table 4 .

Table 4: Output of the descending search.

\begin{tabular}{rrrl}
\hline$n$ & $B_{1}(n)$ & $B_{2}(n)$ & $\left(a_{1}, \ldots, a_{n}\right)$ \\
\hline 18 & & 92 & $(1-7,9-11,13-17,19,23,29)$ \\
19 & 96 & 100 & $(1-3,5,7-13,17,19,23,29,31,41,43,53)$ \\
20 & & 128 & $(1-7,9-11,13,15-17,19::: 29),(1-9,11::: 31)$ \\
22 & & 152 & $(1-11,13::: 31,37)$ \\
27 & & 298 & $(1-4,6-11,13::: 43,47)$ \\
28 & 280 & & $(1-7,9-11,13-17,19,21,23-25,27::: 41)$ \\
28 & & 320 & $(1-7,9-17,19::: 37,41,47)$ \\
29 & 284 & 340 & $(1-7,9-11,13-17,19,21,23-25,27::: 43)$ \\
30 & 328 & & $(1-11,13-17,19,21,23-25,27::: 43)$ \\
30 & & 392 & $(1-15,17::: 43,49)$
\end{tabular}


Table 4: Output of the descending search, continued from the previous page.

\begin{tabular}{|c|c|c|c|}
\hline$n$ & $B_{1}(n)$ & $B_{2}(n)$ & $\left(a_{1}, \ldots, a_{n}\right)$ \\
\hline 31 & 344 & & $(1-17,19::: 43,49)$ \\
\hline 32 & 416 & 500 & $(1-17,19::: 43,47,49)$ \\
\hline 32 & & 500 & $(1-7,9-17,19::: 43,47,49,53)$ \\
\hline 33 & 440 & 520 & $(1-11,13-17,19,21,23-25,27::: 49)$ \\
\hline 37 & 584 & 728 & $(1-17,19::: 55,61)$ \\
\hline 38 & 652 & 852 & $(1-19,21::: 53,57,61)$ \\
\hline 39 & 688 & 856 & $(1-17,19::: 57,61,67)$ \\
\hline 40 & 684 & 840 & $(1-4,4-9,11-13,15-17,19-21,23,25,27-29,31::: 61)$ \\
\hline 40 & 684 & & $(1-17,19::: 61,67)$ \\
\hline 43 & & 1218 & $(1-19,21::: 63,67,71)$ \\
\hline 44 & 976 & 1384 & $(1,2,2-17,19::: 69)$ \\
\hline 45 & 988 & 1400 & $(1,2,2-17,19::: 71)$ \\
\hline 46 & 1136 & 1666 & $(1,2,2-19,21::: 71)$ \\
\hline 47 & 1176 & 1804 & $(1,2,2-19,21::: 73)$ \\
\hline 48 & 1224 & 1800 & $(1-13,15-21,23,25,27-29,31::: 73,79)$ \\
\hline 49 & 1328 & & $(1,2,2-21,23::: 75)$ \\
\hline 49 & & 2088 & $(1-21,23::: 73,79,81)$ \\
\hline 50 & 1448 & 2276 & $(1-21,23::: 75,79,85)$ \\
\hline 52 & 1544 & 2352 & $(1,2,2-23,25::: 77,83)$ \\
\hline 56 & 1840 & 3048 & $(1,2,2-25,27::: 83,89)$ \\
\hline 58 & 2236 & 4154 & $(1,2,2-23,25::: 89,95)$ \\
\hline 59 & 2142 & 3614 & $(1,2,2-25,27::: 89,95)$ \\
\hline 60 & 2336 & 4056 & $(1,2,2-25,27::: 89,93,99)$ \\
\hline 60 & 2336 & & $(1,2,2-27,29::: 89,97)$ \\
\hline 61 & 2570 & 4594 & $(1,2,2-25,27::: 89,93::: 97)$ \\
\hline 64 & 2836 & 5160 & $(1,2,2-25,27::: 99,107)$ \\
\hline 65 & 3078 & 5798 & $(1,2,2-25,27::: 101,107)$ \\
\hline 66 & 2964 & 5428 & $(1,2,2-25,27::: 103,109)$ \\
\hline 67 & 3172 & 5708 & $(1,2,2-13,15-29,31::: 103,109)$ \\
\hline 68 & 3632 & 7340 & $(1,2,2-25,27::: 103,107,109,111)$ \\
\hline 69 & 3888 & 7888 & $(1,2,2-13,15-29,31::: 109)$ \\
\hline 70 & 3904 & 7832 & $(1,2,2-13,15-29,31::: 39,41-43,45::: 109)$ \\
\hline
\end{tabular}


To employ this strategy for determining upper bounds for $A_{1}(n)$ for a fixed value of $n$, one needs starting vectors of length $n+1$. We generated initial vectors by three methods. Firstly, we borrowed Maltby's construction, which is inspired by the theory of root systems. In the notation fixed in Section 1, the exponents $a_{i}$ are obtained from arbitrary natural numbers $d_{j}$ by means of linear relations existing between the positive roots and the simple roots of Weyl groups. Specifically, we looked at the groups of type $A_{t}(5 \leqslant t \leqslant 8), B_{t}$ and $C_{t}$ $(4 \leqslant t \leqslant 6)$, and $F_{4}$. For example, in the case $W=F_{4}$ and $\left(d_{1}, d_{2}, d_{3}, d_{4}\right)=(4,1,8,2)$ one obtains the exponents

$$
\mathbf{a}=(1,2,4,5,8-11,13::: 21,21-26,32,34,39,43,47) .
$$

We found that the exponents generated by this method tend to have 'too many even values' in comparison with the record vectors already available. Indeed, from the last columns of Tables 1-4 one notices that about three-quarters of the entries of the record vectors are odd. Moreover, the even entries are small, and are located in the first half of the vector when this is written in increasing order. In order to comply with this experimental observation, we modified the values produced by Maltby's construction, by replacing some of the even entries by the closest odd integers. The new vectors have repeated entries, while from the available data one may conclude that the record vectors have distinct values (at least for $8 \leqslant n \leqslant 43$ ). So we either ignored multiple values (technically, converting the multisets to sets, which results in shorter vectors) or replaced the even entries by larger odd integers (keeping the length of the vector). Continuing the example given for the Weyl group of type $F_{4}$, we replaced the 24-vector of exponents a by either

$$
(1,2,4,5,8-11,13::: 21,22-25,27,31,33,39,43,47),
$$

or

$$
(1,2,4,5,8-11,13::: 21,22-25,27::: 33,39,43,47) .
$$

We found considerable success with initial vectors obtained by concatenating two previously generated record vectors and removing multiple entries. As a variant of this method, the entries of one of the vectors have been increased by 2, 4, 6, or 8 before concatenation. In this way we obtained longer initial vectors, and consequently upper bounds $B_{1}(n)$ for larger $n$. Occasionally we used random initial vectors, but this idea turned out to be much less successful than the others.

\section{Divisibility conditions}

In this section, we derive some divisibility conditions on the exponents of pure products of minimal $l_{1}$-norm. The idea is to show that the record polynomials must be divisible by certain cyclotomic polynomials $\Phi_{m}(X)$. This has been used by Bombieri and Vaaler [5], Amoroso [1], Boyd [9, 10], and Borwein and Mossinghoff [8] in determining lower bounds on the degree of a polynomial having low height and prescribed vanishing at 1 . The ingredients of the proofs are an explicit formula for the norm of the element $1-\zeta^{t}$ of $\mathbb{Q}(\zeta)$, where $\zeta$ is a primitive $m$ th root of unity, and the upper bounds for $A_{1}(n)$ are as found in the previous sections.

We need the following fact about cyclotomic integers, which appears, for instance, in the proof of [14, Lemma 5].

LEMMA 6.1. Let $m$ and $t$ be positive integers, $d=m / \operatorname{gcd}(m, t)$, and $\zeta$ a primitive $m$ th root of unity. Denote by $N(\alpha)$ the norm of $\alpha \in \mathbb{Q}(\zeta)$, by $\Phi_{m}$ the mth cyclotomic polynomial, and by $\varphi$, Euler's function. Then $N\left(1-\zeta^{t}\right)=\Phi_{d}(1)^{\phi(m) / \phi(d)}$. 
LEMMA 6.2. For each $p$ in the following table, one has $A_{1}(n)<p^{n e(p)}$ for any $n \geqslant n_{0}(p)$.

\begin{tabular}{l|cccccc}
\hline$p$ & 2 & 2 & 3 & 3 & 7 & 11 \\
$e(p)$ & $1 / 2$ & $1 / 4$ & $1 / 4$ & $1 / 6$ & $1 / 6$ & $1 / 6$ \\
$n_{0}(p)$ & 9 & 36 & 15 & 34 & 10 & 20 \\
\hline
\end{tabular}

Proof. Let us show that $A_{1}(n)<2^{n / 2}$ for any $n$ greater than or equal to 9 . For $n \leqslant 17$, the inequality is checked using the upper bounds for $A_{1}(n)$ given in Tables $2-4$. For $n \geqslant 18$, one has $\lfloor n / 2\rfloor \geqslant 9$, so by induction and the submultiplicativity of the $l_{1}$-norm one has

$$
A_{1}(n) \leqslant A_{1}(\lfloor n / 2\rfloor) A_{1}(\lfloor(n+1) / 2\rfloor)<2^{(\lfloor n / 2\rfloor+\lfloor(n+1) / 2\rfloor) / 2}=2^{n / 2} .
$$

Proposition 6.3. For each $m$ in the following table there exists a multiple of $m$ among the exponents giving a product of $n$ binomials whose length is $A_{1}(n)$ for $n \geqslant n_{0}(m)$.

\begin{tabular}{l|rrrrrr}
\hline$m$ & 3 & 4 & 5 & 7 & 8 & 9 \\
$n_{0}(m)$ & 7 & 7 & 7 & 7 & 36 & 34 \\
\hline
\end{tabular}

Proof. Let us prove the claim for $m=9$, for instance. Denote by $P$ a pure product of length $A_{1}(n)$. Let $\zeta$ be a primitive $m$ th root of unity. Since $\Phi_{p^{k}}(1)=p$ for any prime $p$ and any positive integer $k, 3^{n}$ divides $N(P(\zeta))$, by Lemma 6.1. If $m$ does not divide any of the exponents from the binomials giving $P$, then $N(P(\zeta)) \neq 0$, so we have $3^{n} \leqslant|N(P(\zeta))| \leqslant$ $\mathrm{L}(P)^{\varphi(9)}=A_{1}(n)^{6}$. For $n \geqslant n_{0}(m)$, this relation contradicts the inequality for $p=3$, $e_{p}=1 / 6$ given by the previous lemma.

\section{Conclusions}

Theorem 1.2 improves upon Maltby's result, lowering the bound $M$ for the exponents of pure products of length $A_{1}(n)$ from $O\left(n^{0.75 n}\right)$ to $O\left(n^{0.5 n}\right)$. Even this result seems to be over-pessimistic. Based on the data obtained up to now, we conjecture that $A_{1}(n)$ is attained for all $n$ by a product of binomials, all of whose exponents are less than $3^{(n-1) / 2}$. We venture to state a less conservative conjecture: for $n \geqslant 32$, there exist positive integers $a_{1}<a_{2}<\ldots<a_{n}$ of sum $d$ such that $a_{n}$ is smaller than the $\lceil\sqrt{d / 3}\rceil$ th prime number, and the length of the polynomial $\prod_{i=1}^{n}\left(1-X^{a_{i}}\right)$ is $A_{1}(n)$.

The paper describes a way of using several of the many properties apparently shared by the exponents giving $A_{1}(n)$. The precise relationship between the record vectors for consecutive values of $n$ is still undiscovered. We mention a few natural problems suggested by this research.

The large number of odd entries in the record vectors begs an explanation, which would be interesting from a theoretical point of view, and helpful in practice. Explicit computations would benefit by proving that it suffices to search vectors with distinct entries, the smallest of which is 1 or 2. (Similar observations have been made by Borwein and Mossinghoff in [8], prompted by experiments made during their study on polynomials with height 1 and prescribed vanishing at 1.)

The heuristics described in Sections 3-5 work only for small values of $n$. For $n \geqslant 19$, the program implementing conditions (1)-(5) is too time-consuming. The ascending search described in Section 4 is feasible for $n$ up to 60. The method outlined in Section 5 is fast for much higher values of $n$, even in a very poor computational environment. 
Despite its simplicity, the present approach provides better bounds for $A_{1}(n)$ and $A_{2}(n)$ for many values of $n$. We add two more values to those listed in Table 4: the product of binomials with exponents

$$
\text { (1-17, 19-39, } 41::: \text { :51, 53-55, } 57::: 103,107::: 117,123,127,137)
$$

yields $A_{1}(80) \leqslant 7420$ and $A_{2}(80)^{2} \leqslant 21,696$ (improving on the published bound $\left.A_{1}(80) \leqslant 58,488\right)$, and the pure product corresponding to the vector

$$
(1-21,23-39,41-47,49,51-53,55::: 77,79-81 \text {, }
$$

$$
83::: 131,135::: 141,147,151::: 157,167,171)
$$

gives $A_{1}(100) \leqslant 25,048$ and $A_{2}(100)^{2} \leqslant 156,164$ (compare this with $A_{1}(100) \leqslant$ $385,620)$.

The largest $n$ for which bounds for $A_{1}(n)$ and $A_{2}(n)$ can be found in the literature is $n=120$. From [18] we learn that $A_{1}(120) \leqslant 1,440,480$ and $A_{2}(120)^{2} \leqslant 1,025,261,796$. The descending search described in Section 5 produces the example with exponents

$$
\begin{aligned}
& (1-39,41-47,49-63,65::: 69,70,71::: 77,79-81, \\
& 83::: 147,151::: 161,165,167,171,177,181,183,189,197,211),
\end{aligned}
$$

implying that $A_{1}(120) \leqslant 54,244$ and $A_{2}(120)^{2} \leqslant 534,832$. Thus, we have improved on 106 of the 124 values for $A_{1}(n)$ and $A_{2}(n)$ for $n$ in the range $12 \leqslant n \leqslant 70$ and $n=80,100,120$. However, our approach cannot cover the whole search space by itself. It is conceivable that, by providing the values given in Tables 2-4 as the input for Maltby's third algorithm, one could exhaust the search space for small $n$. Then we would have the exact value $A_{1}(n)$, and not just an upper bound $B_{1}(n)$.

Acknowledgements. The author thanks the anonymous referee for several helpful comments.

\section{References}

1. F. Amoroso, 'Polynomials with prescribed vanishing at roots of unity', Boll. Un. Mat. Ital. B (7) 9 (1995) 1021-1042; MR 97a:11120. 46

2. F. V. AtKinson, 'On a problem of Erdős and Szekeres', Canad. Math. Bull. 4 (1961) 7-12; MR 23\# A3722. 39

3. A. S. Belov and S. V. Konyagin, 'On an estimate for the free term of a nonnegative trigonometric polynomial with integer coefficients' (in Russian), Izv. Ross. Akad. Nauk Ser. Mat. 60 (1996) 31-90; translated in Izv. Math. 60 (1996) 1123-1182; MR 99b:42002. 39

4. E. Bombieri and J. VaAler, 'On Siegel's lemma', Invent. Math. 73 (1983) 11-32; MR85g:11049a. 40

5. E. Bombieri and J. VAALER, 'Polynomials with low height and prescribed vanishing', Analytic number theory and Diophantine problems, Progr. Math. 70 (ed. A. C. Adolphson, J. B. Conrey, A. Gosh and R. I. Yager, Birkhäuser, Boston, 1987) 53-73; MR 90k:11133. 46

6. I. BoROsh, 'A sharp bound for positive solutions of homogeneous linear diophantine equations', Proc. Amer. Math. Soc. 60 (1976) 19-21; MR 54:10291. 40 
7. P. Borwein and C. Ingalls, 'The Prouhet-Tarry-Escott problem revisited', L'Enseign. Math. 40 (1994) 3-27; MR 95d:11038. 37, 39, 42

8. P. Borwein and M. J. MossinghofF, 'Polynomials with height 1 and prescribed vanishing at 1', Experiment. Math. 9 (2000) 425-433; MR 2001k:11036. 44, 46, 47

9. D. W. BoYD, 'On a problem of Byrnes concerning polynomials with restricted coefficients, I', Math. Comput. 66 (1997) 1697-1703; MR 98a:11033. 46

10. D. W. Boyd, 'On a problem of Byrnes concerning polynomials with restricted coefficients, II’, Math. Comput. 71 (2002) 1205-1217; MR 2003d:11035. 46

11. M. CIPU, 'The norm of a product of cyclotomic polynomials and the Prouhet-TarryEscott problem' (in Romanian), Proc. Second Yearly Nat. Conf. Romanian Soc. Math. Sci. 1998 (Cluj-Napoca, 1999) 41-44. 39, 40

12. E. Dobrowolski, 'On a question of Lehmer and the number of irreducible factors of a polynomial', Acta Arithm. 34 (1979) 391-401; MR 80i:10040. 39

13. P. Erdő́s and G. SzeKeres, 'On the product $\prod_{k=1}^{n}\left(1-z^{a_{k}}\right)$ ', Acad. Serbe Sci. Publ. Inst. Math. 13 (1959) 29-34; MR 23\#A3721. 38

14. M. Filaseta, 'Coverings of the integers associated with an irreducibility theorem of A. Schinzel', Number theory for the millenium, vol. II (ed. M. A. Bennett, B. C. Berndt, N. Boston, H. G. Diamond, A. J. Hildebrand and W. Philipp, A.K. Peters, Natick, 2002) 1-24. 46

15. M. N. KolountzaKis, 'On non-negative cosine polynomials with non-negative, integral coefficients’, Proc. Amer. Math. Soc. 120 (1994) 157-168; MR 94b:42002. 39

16. R. Maltby, 'Pure product polynomials of small norm', Ph.D. Dissertation, Simon Fraser University, 1996. 39

17. R. Maltby, 'Pure product polynomials and the Prouhet-Tarry-Escott problem', Math. Comp. 66 (1997) 1323-1340; MR 98e:11026. 39, 40, 42

18. R. Maltby, 'Root systems and the Erdős-Szekeres problem', Acta Arithm. 81 (1997) 229-245; MR 99b:11017. 38, 42, 48

19. P. Nowosad and R. Tovar, 'Spectral inequalities and G-functions', Linear Algebra Appl. 31 (1980) 179-197; MR 81g:15021. 40

20. A. M. Odlyzko, 'Minima of cosine sums and maxima of polynomials on the unit circle', J. London Math. Soc. (2) 26 (1982) 412-420; MR 84i:2001. 39

Mihai Cipu mihai.cipu@imar.ro

http://stoilow.imar.ro/ mcipu

Institute of Mathematics of the Romanian Academy

P. O. Box 1-764

RO-014700 Bucharest

Romania 